\title{
The multiple aims of pay-for- performance and the risk of unintended consequences
}

\section{Rocco Friebel, ${ }^{1,2}$ Adam Steventon ${ }^{1}$}

${ }^{1}$ The Health Foundation, London, UK

${ }^{2}$ Imperial College London, London, UK

\section{Correspondence to} Rocco Friebel, Department of Data Analytics, The Health Foundation, 90 Long Acre, London WC2E 9RA, UK; Rocco.Friebel@health.org.uk

Accepted 11 March 2016 Published Online First 5 April 2016

\section{CLlinked}

- http://dx.doi.org/10.1136/ bmjqs-2015-005040

\section{CrossMark}

To cite: Friebel $R$, Steventon A. BMJ Qual Saf 2016:25:827-831.
Since the Affordable Care Act introduced financial penalties on hospitals for excess readmission rates in the USA, an intense debate has ensued regarding the value of readmissions as a marker of quality. Under the Hospital Readmission Reduction Program (HRRP), hospitals face penalties of up to $3 \%$ of base operating payment from Medicare, the federally funded health insurance system for people aged over 65. Penalties totalled $\$ 428$ million in $2015,{ }^{1}$ and similar policies are in place in Denmark, Germany and England. ${ }^{2}$

HRRP aimed to 'reward hospitals that are successful in reducing avoidable readmissions $^{3}$ and indeed Medicare has seen a decline in 30-day, all-cause readmission rates since the policy was introduced in 2012. ${ }^{4}$ More specific declines have been observed for the three conditions initially targeted, namely acute myocardial infarction, heart failure and pneumonia (figure 1). ${ }^{15}$ The HRRP was expanded to cover chronic obstructive pulmonary disease, total hip arthroplasty and total knee arthroplasty from 2015.

While no study has been able to test causality against a counterfactual, on the face of it HRRP has contributed to a sustained focus on readmissions and potentially, improved patient care nationally. But what aspects of quality do readmission rates measure? And what are we to make of the findings reported in this issue of BMJ Quality and Safety, ${ }^{6}$ which indicate that, like other pay-for-performance programmes, readmission penalties have disproportionately affected safety-net hospitals (ie, hospitals that serve a high number of patients of lower socioeconomic status (SES), often uninsured).

\section{THE NATURE OF READMISSION RATES AS A MEASURE OF THE QUALITY OF HEALTHCARE}

In theory, readmissions are related to the quality and safety of the initial hospital stay, the transitional care services and access to care and support following the hospital discharge. Thus, advocates claim, readmissions impose a burden on patients and the healthcare system alike, while being amenable to certain improvements in service delivery. ${ }^{7}$ However, the relationship between readmissions and quality of care is not straightforward, and there are confounding factors, such as regional variations in the propensity of hospitals to admit patients. ${ }^{8}$

The multifactorial nature of readmissions is apparent from analyses that show how rarely they map onto the principal diagnosis from the initial hospital admission. Almost two-thirds of readmissions following a hospitalisation for heart failure are for some other diagnosis $(64.8 \%)$, and figures for acute myocardial infarction and pneumonia are even higher, at $90 \%$ and $78 \%$, respectively. ${ }^{9}$ However, a narrow focus on the acute illness that precipitated the hospitalisation might understate the significance of the allostatic and physiological stress that patients experience while in hospital. Factors such as disruption to sleep, poor nourishment, pain and discomfort, psychological stress, the effect of medications on cognition and physical function and deconditioning might lead to patients being at higher levels of risk in the period immediately following discharge from hospital. ${ }^{10}$ These considerations may explain why HRRP (and indeed readmission policies internationally) tend to focus on all-cause readmissions.

The amenability of readmissions to improvements in care is often unclear, with studies estimating that anywhere between $5 \%$ and $79 \%$ of readmissions are preventable. ${ }^{11} \mathrm{~A}$ linked concern is to what extent readmission rates vary according to factors that are outside of the direct control of hospitals, but which still might be influenced by them. Given 


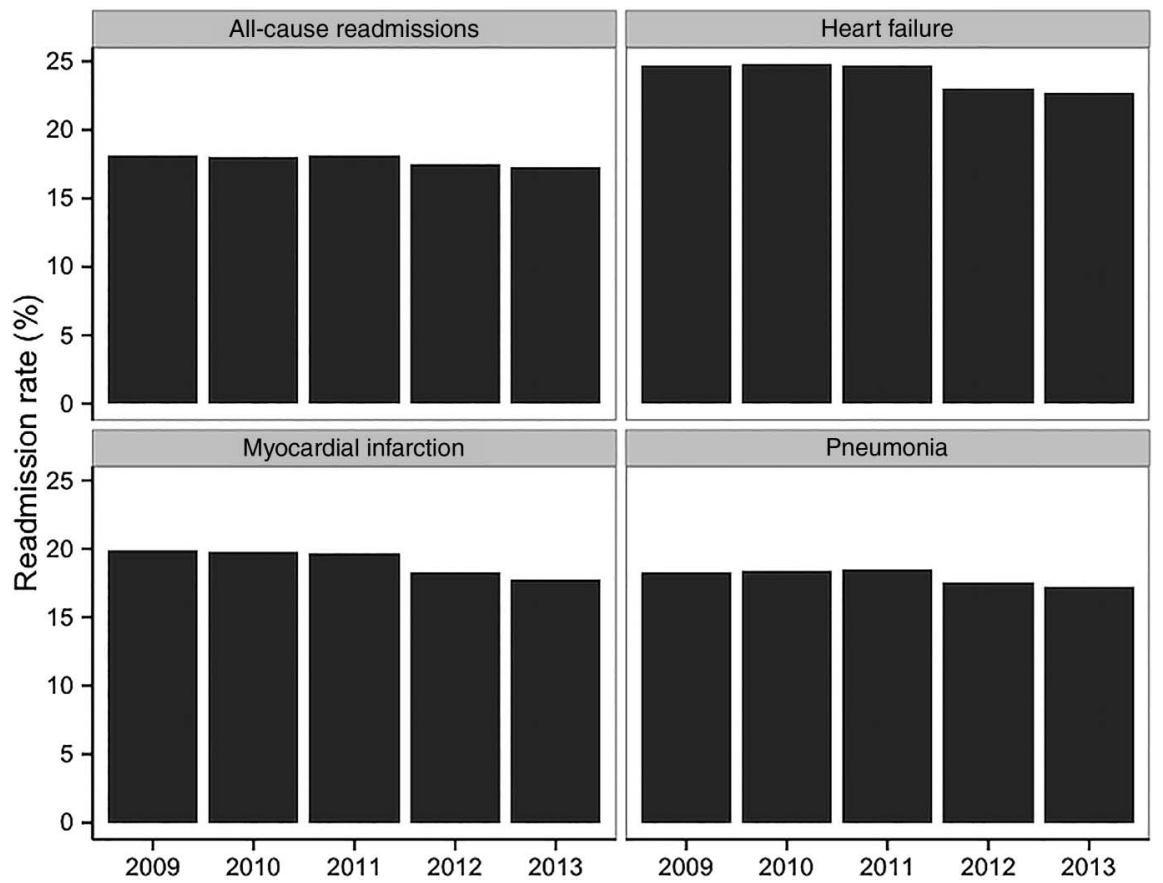

that readmissions are related to care processes at multiple parts of the care pathway, financial penalties on hospitals aim to incentivise improvements within the hospital walls and also to spur co-ordination between primary, ambulatory and secondary care. ${ }^{12}$ Where should the line be drawn? Readmissions are affected by preventive primary care, social care and factors outside of the traditional remit of healthcare, such as social support, income and housing. ${ }^{13}$ To what extent is it reasonable and effective to hold hospitals to account for these aspects?

Risk adjustment is critical to ensuring that hospitals with sicker patients are not unduly penalised for higher readmissions. However, administrative databases contain limited information on healthcare needs, which might explain why studies have produced contradictory findings on the correlation between risk-adjusted hospital readmission rates and risk-adjusted mortality rates. ${ }^{14} 15$

The importance of risk adjustment is illustrated by the paper by Figueroa et al, ${ }^{6}$ which finds that safetynet hospitals were more likely to be penalised under HRRP than other hospitals. Similar patterns were found for two other Medicare pay-for-performance programmes introduced under the Affordable Care Act: ${ }^{16}$ Hospital Value-Based-Purchasing (which targets clinical measures and patient experience) and the Hospital-Acquired Condition Reduction Program. When penalties from the three programmes are combined, safety-net hospitals were more than twice as likely as other hospitals to be in the 'most penalised' group, which on average saw relevant Medicare payments reduced by $1.86 \%$.
While further research is needed to establish more firmly the nature of the relationship between readmissions and quality, in the short term, two questions require urgent attention. First, the paper by Figueroa et $a l^{6}$ and other studies ${ }^{17}$ have identified concerning patterns for safety-net hospitals. Does this mean the risk adjustment should be changed in HRRP? Second, given that the risk adjustment will never be perfect, ${ }^{18}$ what other approaches are available to maximise the potential of pay-for-performance to benefit patients, while reducing the risk of unintended consequences?

\section{Can risk adjustment be improved?}

Following analyses such as those of Figueroa et al, ${ }^{6}$ the main area of contention seems to be the lack of adjustment for SES in the readmission rates used by HRRP. However, the HRRP approach was based on guidance by the National Quality Forum (NQF) and arose from concerns about health inequalities. ${ }^{19}$

People in high-poverty neighbourhoods have a 25\% higher risk of readmission than those in more affluent neighbourhoods in the USA. ${ }^{20}$ These differences might reflect lower quality healthcare and thus adjusting readmission rates for this gradient would effectively set the readmission benchmark less stringently for poorer patients, potentially entrenching health inequalities.

Given the findings reported for safety-net hospitals, a natural response is to revisit the original NQF decision and argue that readmission metrics should now be adjusted for SES. If the higher readmission rates at safety-net hospitals reflect factors outside of these hospitals' control, then the higher penalties might be 
unfair, especially when they are working proactively to reduce readmissions. ${ }^{21}$ The difficulty is that it is unclear whether higher readmission rates for lower SES patients reflect poorer quality of hospital care or factors beyond the hospital's direct control or influence (eg, poor access to primary care). In reality, residents of socioeconomically deprived areas tend to face multiple disadvantages, possibly experiencing both lower quality hospital stays and lower access to ambulatory care. Hence, there is no simple solution with respect to the risk adjustment for SES.

Although studies have shown that readmission rates are related to SES at the patient or hospital level, ${ }^{22} 23$ these cannot be taken to imply direct causality between SES and observed differences. Teasing out issues of healthcare need and supply is notoriously difficult. $^{24}$ The relationship might be confounded by a range of factors, the quality of healthcare and also admission thresholds, unmeasured aspects of SES and patient characteristics. Currently available data sets do not allow these factors to be isolated.

A technical report published by NQF in 2014 recommended a general shift towards adjusting outcome measures for SES, although on a case-by-case basis. ${ }^{25}$ This has not been taken up by HRRP, but it is unclear to what extent adjusting for SES will change the outlook for safety nets. A recent study from New York State examined readmissions following six major surgical interventions and found that $51 \%$ of safety-net hospitals had greater than predicted readmissions with SES adjustment, compared with 61\% without SES adjustment (figure 2). ${ }^{26}$ But studies for heart failure have found that SES adjustment very rarely changes whether or not a hospital is ranked worse than average. ${ }^{27} 28$ Additional research is urgently needed to examine national data across all the conditions targeted by HRRP. Studies could also investigate to what extent the impact of risk adjusting for SES depends on the particular algorithm used to calculate the penalties. HRRP works by comparing readmission rates at the hospital level, but the New York study showed that the odds ratio (OR) for readmission could fall by a greater amount if this calculation was done at patient level (in this instance, the OR for safety-net hospitals fell from $14 \%$ to $8 \%$ with SES adjustment).

While technical advances to risk adjustment should be pursued, it must be emphasised that, due to limitations of methodology and data, there is no single 'right answer'. The danger is that too narrow a focus places unreasonable expectations on risk adjustment to resolve the underlying issues. Other approaches are available, including some borrowed from quality improvement, like logic models and measurement frameworks. ${ }^{29}$

\section{What else can be done?}

Attempts to improve healthcare using data almost always involve a shuttling back and forth between the aims, theory of change and metrics. The findings of Figueroa $e t a l^{6}$ highlight a concerning pattern for safety-net hospitals. What remains unclear is whether the response should be restricted to further evolution of the metrics as the authors suggest, or whether the

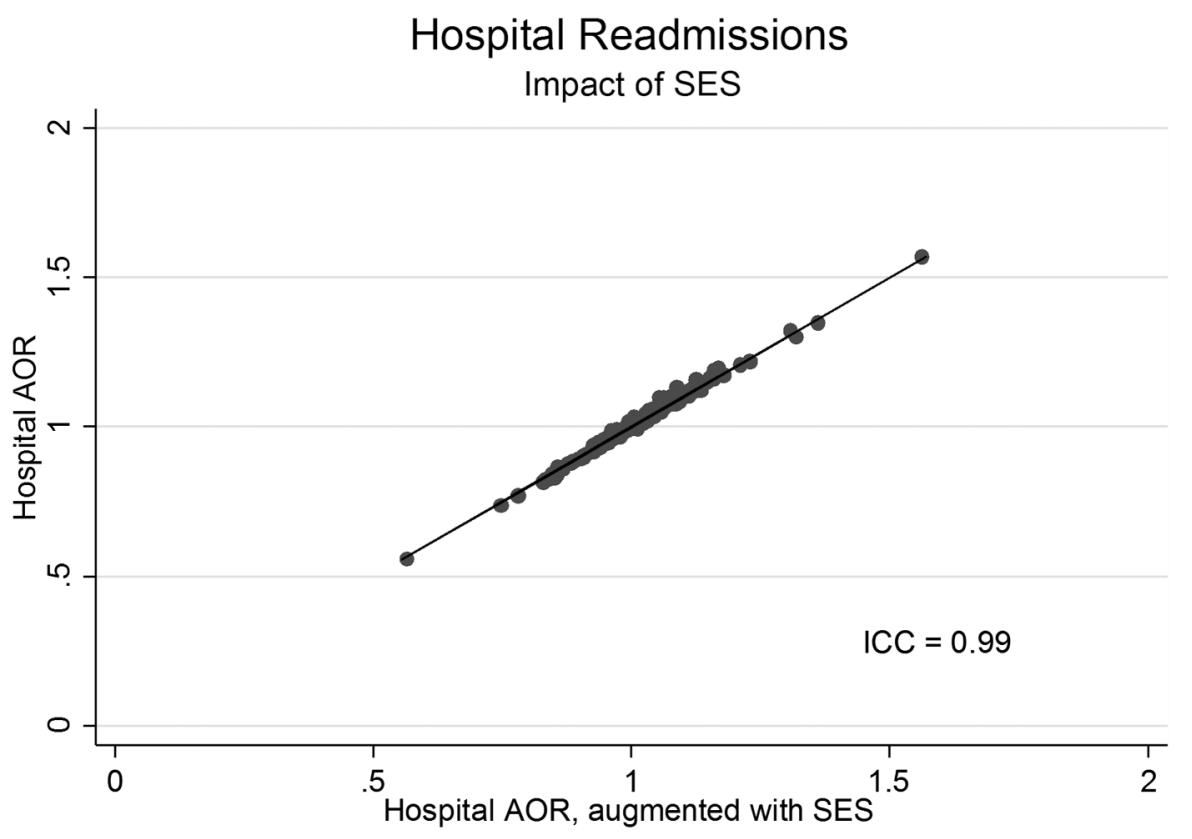

Figure 2 Impact of socioeconomic status (SES) risk adjustment on 30-day readmission rates. ${ }^{26}$ The y-axis shows hospitals' risk-adjusted readmission rates for surgery, under the current Hospital Readmission Reduction Program (HRRP) approach, which does not include risk adjustment for SES. The x-axis shows rates that are additionally adjusted for SES. 
aims of the policy and theory of change should also be updated following their analysis.

The aims of any initiative often evolve in response to changes in context. It is now possible to discern several potential goals for HRRP besides the one to reduce readmissions, and these may include equity between providers, the financial sustainability of safety-net hospitals, addressing health inequalities and better integration of healthcare between traditional secondary care and community settings. To what extent are readmission penalties intended to address these aims, how should they be traded off against each other and how much risk is tolerable? Answers to these questions can be informed by the outcomes of other initiatives to address integration (eg, patientcentred medical homes, accountable care organisations) and health inequalities (eg, Medicaid expansion, insurance reforms and initiatives such as Healthy People 2020). ${ }^{30}$

Given that any policy has multiple aims, what array of metrics will give decision makers the best chance of monitoring progress and making course correction when needed? A system of financial penalties will always emphasise a small number of metrics that are linked to payment. But a series of metrics is required given the potential for readmission rates to correlate with several quality domains, and the risk of unintended consequences from HRRP, as might result, for example, if safety-net hospitals were relatively underfunded.

The scientific literature on readmissions is growing rapidly. The paper by Figueroa et $a l^{6}$ powerfully illustrates the value of descriptive analysis, but we would caution against a narrow response to these findings focused solely on changing the risk adjustment. The multiple aims of pay-for-performance must be surfaced, and a measurement framework designed and put in place that helps us reach our goals.

Acknowledgements We thank Harlan Krumholz, Kumar Dharmarajan and Will Warburton for providing valuable comments on a previous version of the manuscript. This article represents independent research supported by the National Institute for Health Research (NIHR) Imperial Patient Safety Translational Research Centre. The views expressed are those of the authors and not necessarily those of the NHS, the NIHR or the Department of Health.

Competing interests None declared.

Provenance and peer review Commissioned; internally peer reviewed.

\section{REFERENCES}

1 Boccuti C, Casillas G. Aiming for Fewer Hospital U-turns: The Medicare Hospital Readmission Reduction Program. Policy Brief. 2015. http://bit.ly/1KWsOkd

2 Kristensen SR, Bech M, Quentin W. A roadmap for comparing readmission policies with application to Denmark, England, Germany and the United States. Health Policy 2015;119:264-73.

3 Center for Medicare and Medicaid. Affordable Care Act Update: Implementing Medicare Cost Savings. 2010. https:// www.cms.gov/apps/docs/aca-update-implementingmedicare-costs-savings.pdf

4 Barrett ML, Wier LM, Jiang J, et al. All-cause readmissions by payer and age, 2009-2013. Agency for Healthcare Research and Quality, Rockville, MD, 2015.

5 Zuckerman RB, Sheingold SH, Orav EJ, et al. Readmissions, observation, and the hospital readmissions reduction program. N Engl J Med 2016; doi:10.1056/NEJMsa1513024

6 Figueroa JF, Wang DE, Jha AK. Characteristics of hospitals receiving the largest penalties by US pay-for-performance programmes. BMJ Qual Saf 2016;25:898-900.

7 Dharmarajan K. Comprehensive strategies to reduce readmissions in older patients with cardiovascular disease. Can J Cardiol. Published Online First: 2016; doi:10.1016/j.cjca.2016.01.030

8 Bhatia RS, Austin PC, Stukel TA, et al. Outcomes in patients with heart failure treated in hospitals with varying admission rates: population-based cohort study. BMJ Qual Saf 2014;23:981-8.

9 Dharmarajan K, Hsieh AF, Lin Z, et al. Diagnoses and timing of 30-day readmissions after hospitalization for heart failure, acute myocardial infarction, or pneumonia. JAMA 2013;309:355-63.

10 Krumholz HM. Post-hospital syndrome-an acquired, transient condition of generalized risk. N Engl J Med 2013;368:100-2.

11 van Walraven C, Jennings A, Taljaard M, et al. Incidence of potentially avoidable urgent readmissions and their relation to all-cause urgent readmissions. CMAJ 2011;183:1067-72.

12 American College of Emergency Physicians. Medicare's Hospital Readmission Reduction Program FAQ. 2015. http:// www.acep.org/Physician-Resources/Practice-Resources/ Administration/Financial-Issues-/-Reimbursement/ Medicare-s-Hospital-Readmission-Reduction-Program-FAQ/

13 Marmot M, Wilkinson RG. Social determinants of health. 2nd edn. Oxford: Oxford University Press, 2005.

14 Laudicella M, Li Donni P, Smith PC. Hospital readmission rates: signal of failure or success? J Health Econ 2013;32:909-21.

15 Krumholz HM, Lin Z, Keenan PS, et al. Relationship between hospital readmission and mortality rates for patients hospitalized with acute myocardial infarction, heart failure, or pneumonia. JAMA 2013;309:587-93.

16 Strokoff SL, Grossman EG. Compilation of Patient Protection and Affordable Care Act. 2010. http://www.hhs.gov/healthcare/ rights/law/

17 Joynt KE, Orav EJ, Jha AK. Thirty-day readmission rates for Medicare beneficiaries by race and site of care. JAMA 2011;305:675-81.

18 Marang-van de Mheen PJ, Shojania KG. Simpson's paradox: how performance measurement can fail even with perfect risk adjustment. BMJ Qual Saf 2014;23:701-5.

19 Fiscella K, Burstin HR, Nerenz DR. Quality measures and sociodemographic risk factors: to adjust or not to adjust. JAMA 2014;312:2615-16.

$20 \mathrm{Hu}$ J, Gonsahn MD, Nerenz DR. Socioeconomic status and readmissions: evidence from an urban teaching hospital. Health Aff 2014;33:778-85.

21 Amarasingham R, Patel PC, Toto K, et al. Allocating scarce resources in real-time to reduce heart failure readmissions: a prospective, controlled study. BMJ Qual Saf 2013;22:998-1005.

22 Damiani G, Salvatori E, Silvestrini G, et al. Influence of socioeconomic factors on hospital readmissions for heart failure and acute myocardial infarction in patients 65 years and older: Evidence from a systematic review. Clin Interv Aging 2015;10:237-45. 
23 Calvillo-King L, Arnold D, Eubank KJ, et al. Impact of social factors on risk of readmission or mortality in pneumonia and heart failure: systematic review. J Gen Intern Med 2013;28:269-82.

24 Wennberg JE, Staiger DO, Sharp SM, et al. Observational intensity bias associated with illness adjustment: cross sectional analysis of insurance claims. BMJ 2013;346:f549.

25 National Quality Forum. Risk adjustment for sociodemographic factors. 2014. http://www.qualityforum.org/risk_adjustment_ ses.aspx

26 Glance LG, Kellermann AL, Osler TM, et al. Impact of risk adjustment for socioeconomic status on risk-adjusted surgical readmission rates. Ann Surg 2016;263:698-704.
27 Blum AB, Egorova NN, Sosunov EA, et al. Impact of socioeconomic status measures on hospital profiling in New York City. Circ Cardiovasc Qual Outcomes 2014;7:391-7.

28 Eapen ZJ, McCoy LA, Fonarow GC, et al. Utility of Socioeconomic Status in Predicting 30-Day Outcomes after Heart Failure Hospitalization. Circ Hear Fail 2015;8:473-80.

29 Simpson M. A quality improvement plan to reduce 30-day readmissions of heart failure patients. J Nurs Care Qual 2014;29:280-6.

30 Healthy People 2020: An Opportunity to Address Societal Determinants of Health in the United States. U.S. Dep. Heal. Hum. Serv. 2010. http://www.healthypeople.gov/2010/hp2020/ advisory/SocietalDeterminantsHealth.htm 